\title{
Comparison of Attention-Deficit/Hyperactivity Disorder Practice in Adults According to a Training Background in Child Psychiatry
}

\author{
Minha Hong ${ }^{1}$, Seung-Yup Lee ${ }^{2}$, Young Sik Lee ${ }^{3}$, Bongseog Kim", \\ Yoo Sook Joung ${ }^{5}$, Hanik K Yoo ${ }^{6}$, Eui-Jung $\mathrm{Kim}^{7}$, Soyoung Irene Lee, \\ Su-Bin Park ${ }^{9}$, Soo-Young Bhang ${ }^{10}$, Doughyun $\mathrm{Han}^{3}$, and Geon Ho Bahn ${ }^{2}$ \\ ${ }^{1}$ Department of Psychiatry, Myongji Hospital, Hanyang University College of Medicine, Seoul, Korea \\ 2Department of Psychiatry, Kyung Hee University School of Medicine, Seoul, Korea \\ ${ }^{3}$ Department of Psychiatry, Chung-Ang University College of Medicine, Seoul, Korea \\ ${ }^{4}$ Department of Psychiatry, Inje University College of Medicine, Seoul, Korea \\ ${ }^{5}$ Department of Psychiatry, Sungkyunkwan University College of Medicine, Seoul, Korea \\ ${ }^{6}$ Seoul Brain Research Institute, Seoul, Korea \\ ${ }^{7}$ Department of Psychiatry, College of Medicine, Ewha Womans University, Seoul, Korea \\ ${ }^{8}$ Department of Psychiatry, Soonchunhyang University College of Medicine, Buchun, Korea \\ ${ }^{9}$ National Center for Mental Health, Seoul, Korea \\ ${ }^{10}$ Department of Psychiatry, Eulji University Hospital, Eulji University School of Medicine, Seoul, Korea
}

\begin{abstract}
Objectives: Awareness of attention-deficit/hyperactivity disorder (ADHD) in adults has significantly increased; however, clinical data specific to the Korean population are insufficient. Clinical experience of ADHD may differ based on whether psychiatrists have received pediatric psychiatry-specific training. In order to prepare a practice parameter for adult ADHD patients in Korea, we examined questionnaire data to observe how pediatric psychiatry training could affect clinical practice for adults with ADHD.

Methods: A questionnaire about the diagnosis and treatment process was distributed to both general psychiatrists (GPsy) and child and adolescent psychiatrists (CAP) at the summer and winter workshop meetings of Korean Academy of Child and Adolescent Psychiatry.

Results: In total, 142 psychiatrists participated in the survey (86 GPsy, 56 CAP). GPsy and CAP preferred pharmacotherapy (GPsy $82.40 \%$, CAP $64.30 \%$ ) as the primary treatment option and answered that the clinical psychiatric interview is the most necessary step in diagnostic assessment (GPsy 22.16\%, CAP 19.00\%). The GPsy responded with an optimal and average treatment duration that was shorter than that reported by CAP.

Conclusion: Identification of the initial presenting symptom as the correct diagnosis and the optimal duration of pharmacotherapy differed between GPsy and CAP in practice, whereas concepts in diagnosis and treatment of ADHD in adults were similar for both groups. These results suggest the urgent need for the Continuing Medical Education program for psychiatrists treating adults with ADHD.
\end{abstract}

Key Words: Adult; Attention deficit hyperactivity disorder; Practice pattern; Experts.

Received: June 12, 2019 / Revision: June 19, 2019 / Accepted: June 21, 2019

Address for correspondence: Geon Ho Bahn, Department of Psychiatry, Kyung Hee University College of Medicine, 23 Kyungheedae-ro, Dongdaemun-gu, Seoul 02447, Korea

Tel: +82-2-958-8556, Fax: +82-2-957-1997, E-mail: mompeian@khu.ac.kr

\section{INTRODUCTION}

In the Diagnostic and Statistical Manual of Mental Disorders, fifth edition, attention-deficit/hyperactivity disorder (ADHD) is classified under the "neurodevelopmental disorder" section [1]. Persistence of the disorder up to adulthood has been reported in $40-60 \%$ of individuals [2,3]. Although a growing body of research has focused on the persistence of

This is an Open Access article distributed under the terms of the Creative Commons Attribution Non-Commercial License (https://creativecommons.org/licenses/by-nc/4.0) which permits unrestricted non-commercial use, distribution, and reproduction in any medium, provided the original work is properly cited. childhood ADHD through adolescence to adulthood [4,5], professional and public awareness of adult ADHD requires improvement. Concerns associated with stimulant abuse are prevalent, while awareness of the negative consequences resulting from ADHD is highlighted. [6,7] There are no practice guidelines specifically for adult ADHD in Korea, although major guidelines have been published worldwide and expert opinions have been suggested for appropriate treatment for adult ADHD [8,9]. Medical insurance coverage for adult ADHD medication since September 2016, has attributed to the current state of medication, which is inaccurate 
and with an absence of information on the patterns of treatment or compliance. There is a need for the Korean Practice Guideline not only because of issues caused by insurance, but also due to culture specific issues such as the stigma surrounding psychiatric disorders and their medication, differences in drug availability for clinical use, and differences in drug efficacy, and adverse event profiles for Asians, specific for Korean patients. Nonetheless, with the consequent increasing interest in adult ADHD, it has progressively become the main subject of media reports and academic conferences in the field of psychiatry.

Improvement in public awareness has led to an increase in the number of adult ADHD patients seeking general psychiatric specialists as well as child and adolescent psychiatrists (CAP) and general psychiatrists (GPsy), actively involved with treating patients. However, it is our understanding that CAP are more familiar with symptoms and signs of $\mathrm{ADHD}$, due to their experience with childhood and adolescent $\mathrm{ADHD}$, and therefore may be more familiar with the symptoms or signs that change with time. For example, the 'hyperactive' symptom is observed in adults with ADHD as avoidance of symposiums, lectures, or church sermons, while in children, it is observed as standing up while eating or while in the classroom.

In order to establish a practice parameter for adult ADHD, the 'Adult ADHD Study Group in the Korean Academy of Child \& Adolescent Psychiatry' surveyed the expert consensus of GPsy on their diagnostic and treatment methods for adults with ADHD.

\section{METHODS}

Prior to the survey, the use of the questionnaire was confirmed through the several meetings with authors who were members of Adult ADHD Study Group. The survey was conducted with the voluntary participation of Korean psychiatrists.

In Korea, one can become a board-certified psychiatrist (GPsy) after passing the 4-year psychiatric resident training exam and then a qualified CAP by undergoing an additional 2-year training. Here, we categorized the psychiatrists without child and adolescent psychiatric training as GPsy, and with child and adolescent psychiatric training as CAP.

The survey questionnaire was distributed at the summer, August 2018, and winter, February 2019, workshops of Korean Academy of child and adolescent psychiatry from August 2018 to February 2019. Among the 195 attendants, responses were received from 142 members: 86 GPsy and 56 CAP. The response rate was $74.3 \%$.

The questionnaire included the following parameters; 1 ) sociodemographic variables such as age, sex, and years of clinical experience, 2) opinion and clinical practice about diagnostic assessment of adult ADHD such as chief complaints at initial visit and steps to diagnosis and differential diagnosis, and 3) opinion and clinical practice about adult ADHD treatment, such as preferred treatment options, preference of anti-ADHD medication, and treatment duration.

\section{Data analysis}

Descriptive statistics were calculated to analyze demographic characteristics. We used Student's t-test to compare the means and the Chi-squared $\left(\chi^{2}\right)$ test to compare proportions. We did not include missing responses in our computations. The data were analyzed using SPSS for Windows (version 21.0; IBM Corp., Armonk, NY, USA).

\section{Ethics statement}

This study's protocol was approved by the Institutional Review Board of Hospital (KMC IRB 2017-02-054).

\section{RESULT}

\section{General}

Psychiatrists in the CAP group were older and had more clinical experience than did those in the GPsy group (Table 1). Percentage of patients showing ADHD core symptoms as the chief complaint at the first clinic visit was statistically different between groups. (GPsy 48.16\%, CAP 56.48; $\mathrm{p}=0.045$ ). The presenting symptoms of bipolar disorder, anxiety disorder, and substance abuse disorders (SUDs) as the chief complaints in patients with ADHD were significantly higher in the GPsy group ( $\mathrm{p}=0.016, \mathrm{p}=0.011, \mathrm{p}=0.004)$ (Table 1).

\section{Diagnosis}

In the diagnostic process, both GPsy and CAP used a selfreport scale, clinical psychiatric interview, psychological tests, and attention tests in order of descending frequencies (Fig. 1).

\section{Treatment}

GPsy and CAP equally prefer pharmacotherapy as the first option, psychoeducation as the second, and cognitive behavioral therapy as the third. (Fig. 2) Optimal treatment duration according to $31.3 \%$ of GPsy and $5.7 \%$ of CAP was less than 1 year. The average duration of treatment in adult ADHD was less than 6 months for 38\% of GPsy and 1.9\% of CAP (Table 2).

\section{DISCUSSION}

Results of this survey suggest that there is no significant difference between GPsy and CAP, with regards to the pref- 
Table 1. Characteristics of respondents of the survey

\begin{tabular}{|c|c|c|c|c|c|}
\hline & $\begin{array}{c}\text { CAP } \\
(n=56)\end{array}$ & $\begin{array}{c}\text { GPsy } \\
(n=86)\end{array}$ & $t / z / \chi^{2}$ & $\mathrm{p}$ & $\begin{array}{c}\text { Total } \\
(\mathrm{n}=142)\end{array}$ \\
\hline \multicolumn{6}{|l|}{ Age (years), n (\%) } \\
\hline Mean & 43.80 & 40.16 & $2.616^{*}$ & 0.010 & 41.57 \\
\hline$<40$ & $22(39.28)$ & $50(58.13)$ & & & $72(50.34)$ \\
\hline $40-50$ & $19(33.92)$ & $22(25.58)$ & & & $41(28.67)$ \\
\hline$\geq 50$ & $13(23.21)$ & $13(15.11)$ & & & $26(18.18)$ \\
\hline No answer & $2(3.57)$ & $1(1.18)$ & & & $3(2.81)$ \\
\hline Sex (male), n (\%) & $24(42.86)$ & $38(44.19)$ & & & $62(43.66)$ \\
\hline $\begin{array}{l}\text { Years of clinical experience, after board } \\
\text { acquisition (mean) }\end{array}$ & 13.62 & 9.99 & $2.542^{*}$ & 0.012 & 11.55 \\
\hline Experience of treatment of adults with ADHD, n (\%) & $55(98.21)$ & $79(91.86)$ & -1.322 & 0.188 & $134(94.37)$ \\
\hline \multicolumn{6}{|l|}{$\begin{array}{l}\text { Number of patients the respondent has experience } \\
\text { with, } n(\%)\end{array}$} \\
\hline$<10$ & $10(18.18)$ & $41(51.90)$ & & & $51(38.06)$ \\
\hline $10-50$ & $31(56.37)$ & $35(44.30)$ & & & $66(49.25)$ \\
\hline$\geq 50$ & $14(25.45)$ & $3(3.80)$ & & & $17(12.69)$ \\
\hline $\begin{array}{l}\text { Percentage of patients showing ADHD core } \\
\text { symptoms as initial clinical presentation } \\
\text { at the 1st visit, \% }\end{array}$ & 56.48 & 48.16 & $2.024^{*}$ & 0.045 & 51.09 \\
\hline \multicolumn{6}{|l|}{ Other than ADHD core symptoms } \\
\hline Bipolar disorder & 14.17 & 23.75 & $-2.465^{*}$ & 0.016 & 20.00 \\
\hline Depressive disorder & 44.48 & 38.39 & 1.541 & 0.126 & 40.57 \\
\hline Anxiety disorder & 23.56 & 32.50 & $-2.602 *$ & 0.011 & 28.37 \\
\hline SUD & 14.04 & 27.17 & $-3.041^{\dagger}$ & 0.004 & 21.31 \\
\hline Personality disorder & 10.33 & 12.14 & -0.285 & 0.800 & 12.11 \\
\hline Intermittent explosive disorder & 25.08 & 24.34 & 0.183 & 0.856 & 24.46 \\
\hline Others & 13.50 & 14.58 & -0.314 & 0.771 & 14.09 \\
\hline $\begin{array}{l}\text { Percentage of patients who were diagnosed } \\
\text { as ADHD, when they were grown up, } \%\end{array}$ & 55.19 & 66.42 & $-1.995^{*}$ & 0.048 & 62.10 \\
\hline
\end{tabular}

erence of a self-report scale and clinical psychiatric interview, while diagnosing adult $\mathrm{ADHD}$, considering the medication, psychoeducation, and cognitive behavioral treatment in adult $\mathrm{ADHD}$.

However, there was a significant difference in GPsy considering comorbid diseases such as bipolar disorder, anxiety disorder, and SUD at the first visit. This suggests that the GPsy interpret the patients' symptoms as a symptom of other diseases common to adults rather than as the core symptoms of ADHD. For a symptom such as "difficulty in concentration," GPsy seem to first consider diagnoses of other conditions that may occur in adulthood and CAP seem to consider ADHD as the first diagnosis in a developmental perspective.

In the absence of a single diagnostic tool that can be called 'gold standard,' a final decision of the clinician based on clinical information is imperative; therefore it is reasonable to conclude that a clinical interview is conducted after the administration of a self-report scale to identify subjective symptoms. Additionally, psychological tests and attention tests, which are comparatively objective and capable of examining attention, are the third and fourth necessary diagnostic assessments. These are performed in order to obtain an objective, evidence-based diagnosis in practice.

In terms of treatment, our result of pharmacotherapy preference as the first option is consistent with the existing guideline that drug treatment should be the first line of treatment unless the person prefers a psychological approach [10].

Average treatment duration and optimal treatment period is significantly shorter for GPsy than CAP, possibly due to the difference of opinion and in clinical experience on longterm course and prognosis.

To date, thorough psychiatric interview is important in diagnosis of adult ADHD [11]. Therefore, clinical experience 
of the physician is important. CAP experience the growth and development of child and adolescent patients with ADHD, while accumulating experience, considering that many parents of child and adolescent patients are with ADHD. With regard to adult $\mathrm{ADHD}$ patients, communication between
GPsy and CAP is essential.

All GPsy need a long time to build up their experience; hence, it is necessary that psychiatrists of the CAP group share their knowledge on the long-term clinical course and their clinical experience. Utilization of valid and appropriate di-

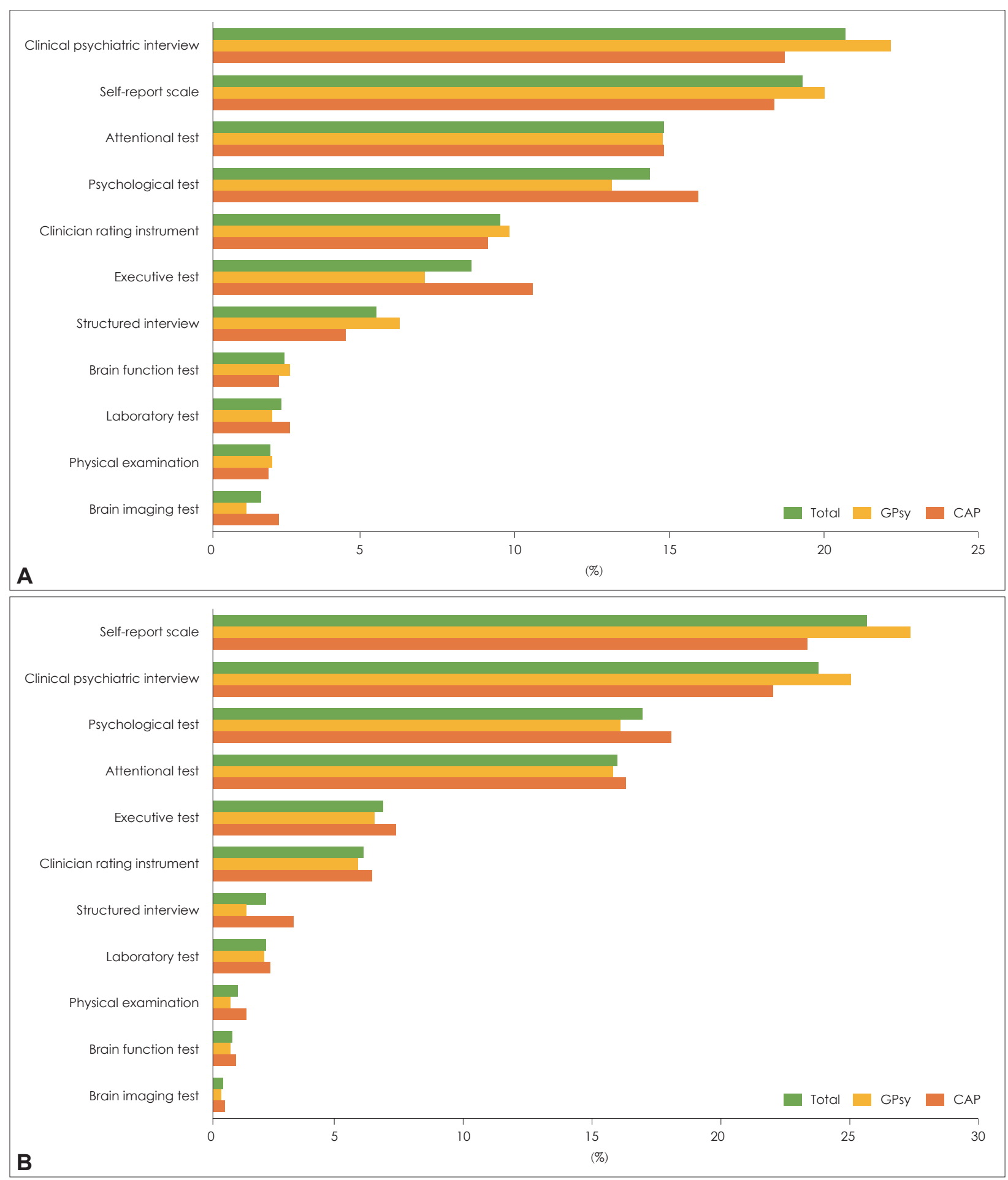

Fig. 1. The diagnostic assessment methods. A: The diagnostic assessment methods in current practice (\%), B: The diagnostic assessment methods in the text-book level knowledge (\%). CAP: child and adolescent psychiatrists, GPsy: general psychiatrists. 


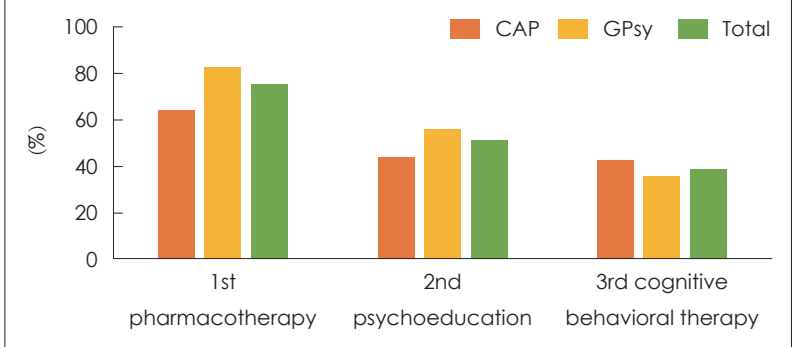

Fig. 2. The preferred treatment options for attention-deficit/hyperactivity disorder in adults. CAP: child and adolescent psychiatrists, GPsy: general psychiatrists.

Table 2. The opinion for the optimal treatment period and average treatment duration

\begin{tabular}{|c|c|c|c|c|c|}
\hline $\begin{array}{l}\text { The optimal } \\
\text { treatment period }\end{array}$ & CAP & GPsy & $\chi^{2}$ & $p$ & Total \\
\hline 6 months or less & $0(0.0)$ & $3(3.8)$ & & & $3(2.3)$ \\
\hline 6 months -1 year & $3(5.7)$ & $22(27.5)$ & & & $25(18.8)$ \\
\hline 2 years & $16(30.2)$ & $18(22.5)$ & $15.078^{*}$ & 0.005 & $34(25.6)$ \\
\hline 3 years and more & $18(34.0)$ & $26(32.5)$ & & & $44(33.1)$ \\
\hline Other & $16(30.2)$ & $11(13.8)$ & & & $27(20.3)$ \\
\hline $\begin{array}{c}\text { The average } \\
\text { treatment duration }\end{array}$ & CAP & GPsy & $\chi^{2}$ & $p$ & Total \\
\hline$\leq 1$ month & $0(0.0)$ & $1(1.3)$ & & & $1(0.8)$ \\
\hline$\leq 3$ months & $1(1.9)$ & $6(7.6)$ & & & $7(5.3)$ \\
\hline$\leq 6$ months & $0(0.0)$ & $23(29.1)$ & & & $23(17.4)$ \\
\hline 6 months -1 year & $27(50.9)$ & $32(40.5)$ & $33.987^{\dagger}$ & 0.000 & $59(44.7)$ \\
\hline 2 years & $12(22.6)$ & $14(17.7)$ & & & $26(19.7)$ \\
\hline$\geq 3$ years & $12(22.6)$ & $1(1.3)$ & & & $13(9.8)$ \\
\hline other & $1(1.9)$ & $2(2.5)$ & & & $3(2.3)$ \\
\hline
\end{tabular}

Data are $n(\%)$ values. ${ }^{*} p<0.05,{ }^{\dagger} p<0.01$. CAP: child and adolescent psychiatrists, GPsy: general psychiatrists

agnostic tools such as Korean ADHD Rating Scale [12,13], which is a screening tool developed by WHO and standardized in Korea, Korean Adult ADHD Rating Scale [12], which has been developed and standardized in Korea, Diagnostic Interview for ADHD in Adults [12,14], which is a semi-structured diagnostic tool for assessing the presence or absence of symptoms by obtaining detailed examples of each item of diagnostic criteria, and Comprehensive Attention Test [12], which has adulthood norms, are needed to complement shortcomings in clinical practice. Current clinical interviews are disadvantaged due to the lack of objectivity, but can be complemented by using the above-mentioned tools when assessing individuals with ADHD.

\section{CONCLUSIONS}

Regardless of whether the psychiatrists receive training for pediatric psychiatry, GPsy have a textbook-level knowledge about ADHD. However, GPsy may not be able to iden- tify and interpret an adult ADHD patient's line of thought. CAP and GPsy may have different indices of suspicion in the initial stage of ADHD diagnosis in adults. Considering the duration of optimal pharmacotherapy, both groups agreed in theory, however, they demonstrated differences in opinions with regards to clinical practice.

Efforts should be made to narrow the gap between the expert opinions of the two groups in order to reduce the potential confusion that may be experienced by patients in clinical practice. As part of this effort, we intend to provide Continuous Medical Education that includes newly developed and updated diagnostic tools and current knowledge on adult $\mathrm{ADHD}$, to improve the knowledge among experts and share clinical experiences to provide more advanced medical care. It is important to offer Continuing Medical Education program to doctors to aid their examination of adult ADHD patients and to collect the data for the long-term follow-up in adults with ADHD.

\section{Conflicts of Interest}

The authors have no potential conflicts of interest to disclose.

\section{REFERENCES}

1) American Psychiatric Association. DSM-5 Task Force. Diagnostic and statistical manual of mental disorders: DSM- $5^{\mathrm{TM}}$. 5th ed. Washington DC: American Psychiatric Association;2013. p.947.

2) Sibley MH, Mitchell JT, Becker SP. Method of adult diagnosis influences estimated persistence of childhood ADHD: a systematic review of longitudinal studies. Lancet Psychiatry 2016;3:1157-1165.

3) Sibley MH, Swanson JM, Arnold LE, Hechtman LT, Owens EB, Stehli A, et al. Defining ADHD symptom persistence in adulthood: optimizing sensitivity and specificity. J Child Psychol Psychiatry 2017;58:655-662.

4) American Psychiatric Association. Diagnostic and statistical manual of mental disorders. 5th ed. Washington DC: American Psychiatric Association Publishing;2013.

5) Agnew-Blais JC, Polanczyk GV, Danese A, Wertz J, Moffitt TE, Arseneault L. Evaluation of the persistence, remission, and emergence of attention-deficit/hyperactivity disorder in young adulthood. JAMA Psychiatry 2016;73:713-720.

6) Caye A, Rocha TB, Anselmi L, Murray J, Menezes AM, Barros FC, et al. Attention-deficit/hyperactivity disorder trajectories from childhood to young adulthood: evidence from a birth cohort supporting a late-onset syndrome. JAMA Psychiatry 2016;73:705-712.

7) Noh GM, Lee SM, Bahn GH. Social function of adult men with attention-deficit/hyperactivity disorder in the context of military service. Neuropsychiatr Dis Treat 2018;14:3349-3354.

8) Kooij SJ, Bejerot S, Blackwell A, Caci H, Casas-Brugué M, Carpentier PJ, et al. European consensus statement on diagnosis and treatment of adult ADHD: The European Network Adult ADHD. BMC Psychiatry 2010;10:67.

9) Young S, Adamou M, Asherson P, Coghill D, Colley B, Gudjonsson $\mathrm{G}$, et al. Recommendations for the transition of patients with ADHD from child to adult healthcare services: a consensus statement from the UK adult ADHD network. BMC Psychiatry 2016;16:301.

10) Faltinsen E, Zwi M, Castells X, Gluud C, Simonsen E, Storebø OJ. Updated 2018 NICE guideline on pharmacological treatments for people with ADHD: a critical look. BMJ Evid Based Med 2019; 
24:99-102.

11) Seixas M, Weiss M, Müller U. Systematic review of national and international guidelines on attention-deficit hyperactivity disorder. J Psychopharmacol 2012;26:753-765.

12) Sibley MH, Pelham WE, Molina BSG, Gnagy EM, Waxmonsky JG, Waschbusch DA, et al. When diagnosing ADHD in young adults emphasize informant reports, DSM items, and impairment.
J Consult Clin Psychol 2012;80:1052-1061.

13) Kim JH, Lee EH, Joung YS. The WHO adult ADHD self-report scale: reliability and validity of the Korean version. Psychiatry Investig 2013;10:41-46.

14) Kooij JJS. Adult ADHD. Diagnostic assessment and treatment. 3rd ed. London: Springer-Verlag;2013. p.292. 